\title{
SUR UN RÉSULTAT D'IMIN CHEN
}

\author{
BART DE SMIT ${ }^{1}$ AND BAS EdIXHOVEN ${ }^{2}$
}

\begin{abstract}
RÉsumÉ. Nous généralisons un résultat de Chen concernant une isogénie entre produits de jacobiennes de courbes modulaires associées à des sous-groupes de $\mathrm{GL}_{2}\left(\mathbb{F}_{p}\right)$. Cette généralisation s'applique à des objets munis d'une action de $\mathrm{GL}_{2}(\mathbb{F})$, avec $\mathbb{F}$ un corps fini quelconque, dans des catégories additives plus générales.
\end{abstract}

RÉSumÉ (VERSION ANGLAise). We generalize a result of Imin Chen concerning an isogeny between products of jacobians of modular curves associated to subgroups of $\mathrm{GL}_{2}\left(\mathbb{F}_{p}\right)$. This generalization concerns objects with an action by $\mathrm{GL}_{2}(\mathbb{F})$, with $\mathbb{F}$ an arbitrary finite field, in more general additive categories.

Le but de cet article est de redémontrer et de généraliser un résultat d'Imin Chen, concernant certaines identités entre fonctions zêta de courbes modulaires, ou, ce qui revient au même, certaines isogénies entre des produits de jacobiennes de telles courbes.

Soient $\mathbb{F}$ un corps fini, $\mathbb{F}^{\prime}$ une extension quadratique de $\mathbb{F}$ et $G:=\mathrm{GL}_{2}(\mathbb{F})$. Le groupe $G$ agit sur $\mathbb{P}^{1}(\mathbb{F})$ et sur $\mathbb{P}^{1}\left(\mathbb{F}^{\prime}\right)$. Soit $B$ le fixateur dans $G$ d'un point $\infty$ dans $\mathbb{P}^{1}(\mathbb{F}), T$ le fixateur dans $G$ de deux points $0, \infty \in \mathbb{P}^{1}(\mathbb{F}), N$ le fixateur de l'ensemble $\{0, \infty\}$, $T^{\prime}$ le fixateur d'un point dans $\mathbb{P}^{1}\left(\mathbb{F}^{\prime}\right)-\mathbb{P}^{1}(\mathbb{F})$, et $N^{\prime}$ le fixateur de sa $\operatorname{Aut}_{\mathbb{F}}\left(\mathbb{F}^{\prime}\right)$-orbite. On appelle $B$ un sous-groupe de Borel, $T$ un tore maximal déployé, et $T^{\prime}$ un tore maximal non déployé. Le groupe $N^{\prime}$ est le normalisateur de $T^{\prime}$ et si $\mathbb{F} \neq \mathbb{F}_{2}, N$ est le normalisateur de $T$.

Rappelons d'abord le résultat de Chen. Pour $n \geq 1$ entier, soit $X(n)_{\mathbb{Q}}$ la courbe modulaire qui est l'espace de modules (grossier si $n<3$ ) compactifié de paires $(E / S / \mathbb{Q}, \phi)$, où $S$ est un $\mathbb{Q}$-schéma, $E / S$ une courbe elliptique et $\phi:(\mathbb{Z} / n \mathbb{Z})^{2} \rightarrow E[n]$ un isomorphisme de $S$-schémas en groupes (voir [10], où ce problème de modules est appelé "naive level $n$ structure"). Par construction, le groupe $\mathrm{GL}_{2}(\mathbb{Z} / n \mathbb{Z})$ agit à droite sur $X(n)_{\mathbb{Q}}$ : un élément $g$ envoie $(E / S / \mathbb{Q}, \phi)$ vers $(E / S / \mathbb{Q}, \phi \circ g)$. Cette action induit, par fonctorialité de Picard, une action à gauche sur la jacobienne de $X(n)_{\mathbb{Q}}$. La jacobienne d'une courbe $X$ sur $\mathbb{Q}$ est

Received November 5, 1999.

1 Boursier du Koninklijke Nederlandse Akademie van Wetenschappen.

2 Membre de l'Institut Universitaire de France, bénéficiaire du European TMR Network Contract ERB FMRX 960006 "arithmetic algebraic geometry". 
un schéma abélien sur $\mathbb{Q}$ notée jac $(X)$. Le résultat de Chen est alors le suivant (voir [2, Theorem 1 et $\S 10]$ ).

Théorème 1 (Chen, 1994). Si $\mathbb{F}=\mathbb{F}_{p}$ avec $p$ premier, $G$ agit sur $X=X(p)_{\mathbb{Q}}$. Alors, $\operatorname{jac}(X / T)$ est isogène à $\operatorname{jac}\left(X / T^{\prime}\right) \times \operatorname{jac}(X / B)^{2}$ et $\operatorname{jac}(X / N)$ est isogène à $\operatorname{jac}\left(X / N^{\prime}\right) \times \operatorname{jac}(X / B)$.

Certains quotients de $X$ sont connus sous d'autres noms (voir [10, Ch. 11], [11, p. 36]):

$$
\begin{aligned}
& X / T \cong X_{0}\left(p^{2}\right)_{\mathbb{Q}}, \quad X / N^{\prime} \cong X(p)_{\text {non-split }} \\
& X / B \cong X_{0}(p)_{\mathbb{Q}}, \quad X / N \cong X_{0}\left(p^{2}\right)_{\mathbb{Q}} /\left\langle w_{p^{2}}\right\rangle .
\end{aligned}
$$

La preuve donnée par Chen consiste à montrer que les traces des opérateurs de Hecke $T_{n}$ avec $n$ premier à $p$ sur les jacobiennes en question satisfont les identités requises pour conclure, par la relation d'Eichler et Shimura et par la conjecture de Tate (démontrée par Faltings), à l'existence d'une telle isogénie. Une preuve n'utilisant que la théorie des représentations de $G$ a été donnée dans [6]; cette note améliore la méthode de [6], et donne quelques résultats supplémentaires (Théorèmes 3 et 4). Voir [5] pour des applications aux variantes de l'équation de Fermat. Dans [1] on trouve une preuve, inspirée par [6] et par l'interprétation adélique des formes modulaires.

Pour un groupe $H$ et $M$ un objet muni d'une action de $H$ dans une catégorie $\mathcal{C}$ nous dirons que $M$ admet des invariants par $H$ si le foncteur $\operatorname{Hom}(-, M)^{H}$ est représentable. Dans ce cas, nous notons $M^{H}$ le sous-objet de $M$ représentant ce foncteur.

Théorème 2. Soit $\mathcal{C}$ une catégorie additive et $\mathbb{Q}$-linéaire. Soit $M$ un objet de $\mathcal{C}$ muni d'une action $\alpha$ de $G$, qui admet des invariants par les sous-groupes de $G$. Alors il existe des isomorphismes fonctoriels en $(M, \alpha)$ :

$$
\begin{aligned}
M^{T} \oplus M^{G} \oplus M^{G} & \cong M^{T^{\prime}} \oplus M^{B} \oplus M^{B} \\
M^{N} \oplus M^{G} & \cong M^{N^{\prime}} \oplus M^{B} .
\end{aligned}
$$

Pour voir que le Théorème 1 est un cas spécial, on applique le Théorème 2 avec $\mathcal{C}=\mathbb{Q} \otimes \mathcal{A}$, où $\mathcal{A}$ est la catégorie des variétés abéliennes sur $\mathbb{Q}$. Les objets de $\mathcal{C}$ sont donc les mêmes que ceux de $\mathcal{A}$, et on a, pour deux objets $A$ et $B$, $\operatorname{Hom}_{\mathcal{C}}(A, B)=\mathbb{Q} \otimes \operatorname{Hom}_{\mathcal{A}}(A, B)$. Pour un objet $A$ de $\mathcal{A}$ nous noterons $\mathbb{Q} \otimes A$ l'objet correspondant de $\mathcal{C}$. Par construction, $A$ et $B$ dans $\mathcal{A}$ sont isogènes si et seulement si $\mathbb{Q} \otimes A$ et $\mathbb{Q} \otimes B$ sont isomorphes dans $\mathcal{C}$. La catégorie $\mathcal{A}$ est abélienne (donc les objets des invariants existent), $\mathbb{Q}$-linéaire et même semisimple. Soit maintenant $M:=\mathbb{Q} \otimes \operatorname{jac}(X)$. Pour tout sous-groupe $H$ de $G$ on a alors $M^{H}=\mathbb{Q} \otimes \operatorname{jac}(X / H)$. On a $M^{G}=0$ car $X / G$ est de genre zéro. 
Preuve du Théorème 2. Par le lemme de Yoneda (voir [7, Ch. 0, §1]), on se ramène au cas où $\mathcal{C}$ est la catégorie des $\mathbb{Q}$-espaces vectoriels. Notons, pour $H \subset G$ un sous-groupe, $\mathbb{Q}[G / H]$ le $\mathbb{Q}[G]$-module donné par l'action de multiplication à gauche de $G$ sur $G / H$; c'est l'induite à $G$ de la représentation triviale de $H$. Avec ces notations, on a, pour tout $\mathbb{Q}[G]$-module $M$ :

$$
M^{H}=\operatorname{Hom}_{\mathbb{Q}[H]}(\mathbb{Q}, M)=\operatorname{Hom}_{\mathbb{Q}[G]}(\mathbb{Q}[G / H], M) .
$$

Il suffit donc de montrer qu'il existe des isomorphismes de $\mathbb{Q}[G]$-modules:

$$
\begin{aligned}
\mathbb{Q}[G / T] \oplus \mathbb{Q} \oplus \mathbb{Q} & \cong \mathbb{Q}\left[G / T^{\prime}\right] \oplus \mathbb{Q}[G / B] \oplus \mathbb{Q}[G / B] ; \\
\mathbb{Q}[G / N] \oplus \mathbb{Q} & \cong \mathbb{Q}\left[G / N^{\prime}\right] \oplus \mathbb{Q}[G / B] .
\end{aligned}
$$

D'après $[14, \S 12]$, il suffit, pour montrer que deux $\mathbb{Q}[G]$-modules de type fini sont isomorphes, de vérifier que leurs caractères coïncident. Dans notre cas, tout élément de $G$ a un conjugué dans $B$ ou dans $T^{\prime}$. Pour montrer l'existence du premier isomorphisme, nous allons montrer que les $G$-ensembles

$$
X=G / T \sqcup\{\cdot\} \sqcup\{\cdot\}, \quad Y=G / T^{\prime} \sqcup G / B \sqcup G / B,
$$

sont isomorphes en tant que $B$-ensembles et en tant que $T^{\prime}$-ensembles. Par la définition de $T, T^{\prime}$ et $B$ on a:

$$
X=\left(\mathbb{P}^{1}(\mathbb{F}) \times \mathbb{P}^{1}(\mathbb{F})-\Delta\right) \sqcup\{\cdot\} \sqcup\{\cdot\}, \quad Y=\mathbb{P}^{1}\left(\mathbb{F}^{\prime}\right) \sqcup \mathbb{P}^{1}(\mathbb{F}) .
$$

Notons $\sigma$ l'élément non trivial de $\operatorname{Aut}_{\mathbb{F}}\left(\mathbb{F}^{\prime}\right)$. En tant que $B$-ensemble, $\mathbb{P}^{1}(\mathbb{F})$ est la réunion disjointe de la droite affine $\mathbb{F}$ et d'un point $\infty$. Comme l'action de $B / \mathbb{F}^{*}$ sur $\mathbb{F}$ est simplement doublement transitive, on a un isomorphisme de $B$-ensembles:

$$
X \cong B / \mathbb{F}^{*} \sqcup \mathbb{F} \sqcup \mathbb{F} \sqcup\{\cdot\} \sqcup\{\cdot\} .
$$

D'autre part, $\mathbb{P}^{1}\left(\mathbb{F}^{\prime}\right)-\mathbb{P}^{1}(\mathbb{F})$ est un $B / \mathbb{F}^{*}$-ensemble libre, car si un élément de $B$ en fixe $P$, il fixe les trois points $P, \sigma(P)$ et $\infty$ de $\mathbb{P}^{1}\left(\mathbb{F}^{\prime}\right)$, donc il est scalaire. Ceci achève la preuve que $X$ et $Y$ sont isomorphes en tant que $B$-ensembles. Considérons maintenant $X$ et $Y$ comme $T^{\prime}$-ensembles. Par définition, $T^{\prime}$ fixe deux points conjugués de $\mathbb{P}^{1}\left(\mathbb{F}^{\prime}\right)$, donc tout élément de $T^{\prime}$ qui fixe un troisième point de $\mathbb{P}^{1}\left(\mathbb{F}^{\prime}\right)$ est scalaire. Il en résulte que $X$ et $Y$ ont chacun deux points fixes par $T^{\prime}$, dont les compléments sont des $T^{\prime} / \mathbb{F}^{*}$-ensembles libres. Comme $\# X=\# Y$, on a $X \cong Y$ en tant que $T^{\prime}$-ensembles, ce qui termine la preuve de l'existence d'un isomorphisme (3).

Pour établir (4), il suffit de montrer que les $G$-ensembles:

$$
\bar{X}:=\left(\operatorname{Sym}^{2}\left(\mathbb{P}^{1}(\mathbb{F})\right)-\Delta\right) \sqcup\{\cdot\}, \quad \bar{Y}:=\mathbb{P}^{1}\left(\mathbb{F}^{\prime}\right) /\langle\sigma\rangle,
$$

sont isomorphes en tant que $B$-ensembles et $T^{\prime}$-ensembles. Comme $\bar{X}$ et $\bar{Y}$ sont quotients de $X$ et $Y$, on voit que les $B$-ensembles $\bar{X}$ et $\bar{Y}$ sont de la forme: $B / H \sqcup \mathbb{F} \sqcup\{\cdot\}$, avec $H$ contenant $\mathbb{F}^{*}$ d'indice 2 . Un tel $H$ est unique à conjugaison près dans $B$, donc $\bar{X} \cong \bar{Y}$ en tant que $B$-ensembles. 
Comme $T^{\prime}$ est cyclique, deux $T^{\prime}$-ensembles transitifs sont isomorphes si et seulement si ils ont même cardinal. On a $\# \bar{X}=\# \bar{Y}$. Alors, pour voir que $\bar{X}$ et $\bar{Y}$ sont $T^{\prime}$-isomorphes, il suffit de montrer qu'il sont tous les deux $T^{\prime} / \mathbb{F}^{*}$-libres, à un point et à au plus une autre orbite près. Supposons qu'un $t$ non trivial dans $T^{\prime} / \mathbb{F}^{*}$ fixe un élément $\{P, Q\}$ de $\operatorname{Sym}^{2}\left(\mathbb{P}^{1}(\mathbb{F})\right)-\Delta$. Alors $t^{2}$ fixe quatre points dans $\mathbb{P}^{1}\left(\mathbb{F}^{\prime}\right)$, donc est scalaire. Il existe au plus un tel $t$ et on a $Q=t P$. Comme $T^{\prime}$ agit transitivement sur $\mathbb{P}^{1}(\mathbb{F})$, cela fait au plus une orbite non $T^{\prime} / \mathbb{F}^{*}$-libre dans $\operatorname{Sym}^{2}\left(\mathbb{P}^{1}(\mathbb{F})\right)-\Delta$. Notons $0^{\prime}$ et $\infty^{\prime}$ les deux points fixes de $T^{\prime}$ dans $\mathbb{P}^{1}\left(\mathbb{F}^{\prime}\right)$. L'action de $T^{\prime}$ sur la $\mathbb{F}^{\prime}$-droite $0^{\prime}$ dans $\mathbb{F}^{\prime 2}$ est un isomorphisme de $T^{\prime}$ vers $\mathbb{F}^{\prime *}$; dès maintenant, nous verrons les $T^{\prime}$-ensembles comme des $\mathbb{F}^{\prime *}$-ensembles via cet isomorphisme. L'action de $\mathbb{F}^{\prime *}$ sur la droite $\infty^{\prime}$ est donnée par $\sigma$. Soit $e_{1}$ un élément non nul de $0^{\prime}$, et $e_{2}:=\sigma\left(e_{1}\right)$ son image dans $\infty^{\prime}$. Cette base de $\mathbb{F}^{\prime 2}$ donne une bijection entre $\mathbb{P}^{1}\left(\mathbb{F}^{\prime}\right)-\left\{0^{\prime}, \infty^{\prime}\right\}$ et $\mathbb{F}^{\prime *}$, qui envoie la droite $\mathbb{F}^{\prime}\left(e_{1}+a e_{2}\right)$ à $a \in \mathbb{F}^{\prime *}$. Via cette bijection, $\sigma$ sur $\mathbb{P}^{1}\left(\mathbb{F}^{\prime}\right)-\left\{0^{\prime}, \infty^{\prime}\right\}$ correspond à $\phi: z \mapsto 1 / \sigma(z)$ sur $\mathbb{F}^{\prime *}$. De même, pour $t$ dans $\mathbb{F}^{\prime *}$, l'action de $t$ devient $z \mapsto(\sigma(t) / t) z$. Les orbites de $T^{\prime}$ sur $\mathbb{F}^{\prime *}$ sont les fibres de la norme $\mathbb{F}^{\prime *} \rightarrow \mathbb{F}^{*}$, tandis que $\phi$ agit sur l'ensemble de ces fibres par inversion de la norme. L'action de $\phi$ sur l'orbite des éléments de norme 1 est triviale, donc donne une orbite $T^{\prime} / \mathbb{F}^{*}$-libre dans $\bar{Y}$, ainsi que les orbites de normes autres que 1 et -1 .

Remarque 1. Nous avons formulé le Théorème 2 pour des objets avec action de $G$ dans des catégories additives $\mathbb{Q}$-linéaires admettant des invariants, pour pouvoir l'appliquer également aux motifs de Chow, par exemple ceux associés aux espaces de formes modulaires de poids au moins deux, construits comme dans [12], ou dans d'autres catégories $\mathbb{Q}$-linéaires pseudo-abéliennes. Rappelons (par exemple [13, §1]) qu'une catégorie additive est dite pseudo-abélienne si pour tout objet $M$ tout idempotent dans $\operatorname{End}(M)$ admet un noyau (ou, ce qui est équivalent, une image). Pour un groupe fini $H$ opérant sur un objet $M$ l'objet $M^{H}$ est l'image de l'idempotent $(1 / \# H) \sum_{h} h$.

Remarque 2. L'idée d'utiliser la théorie des représentations d'un groupe fini $G$ pour en déduire des isogénies entre variétés abéliennes n'est certainement pas nouvelle; voir [8] et [9]. La preuve du Théorème 2 montre que ce genre de résultats reste vrai dans le cadre plus général des catégories additives $\mathbb{Q}$-linéaires pseudo-abéliennes. Dans $[8, \S 5]$ on trouve une relation pour certains sous-groupes de $\mathrm{SL}_{2}\left(\mathbb{F}_{p}\right) /\{1,-1\}$, mais pas les relations du Théorème 2 . L'intérêt du résultat de Chen est que l'on obtient des renseignements sur la jacobienne de $X(p)_{\text {non-split }}$ en termes d'objets déjà mieux compris.

Remarque 3. Loïc Merel a posé la question de savoir si la correspondance constituée des deux morphismes quotients $X \rightarrow X / N^{\prime}$ et $X \rightarrow X / N$ induit un morphisme de la jacobienne de $X / N^{\prime}$ vers celle de $X / N$ dont le noyau est fini. La réponse dépend de la position relative des sous-groupes $N$ et $N^{\prime}$ dans $G$. En 
effet, à conjugaison près, il existe un unique couple $\left(T, T^{\prime}\right)$ avec $T$ déployé et $T^{\prime}$ non déployé, telle que $N \cap N^{\prime}$ soit de cardinal $4(p-1)$. Pour cette configuration, Chen a montré que le noyau en question est fini; voir [3].

Nous nous intéressons maintenant à la question de savoir quels dénominateurs sont essentiels dans le Théorème 2. Plus précisément, on veut remplacer la condition "Q্-linéaire" sur la catégorie $\mathcal{C}$ par " $\mathbb{Z}_{(l)}$-linéaire" avec $l$ premier et $\mathbb{Z}_{(l)}$ le localisé de $\mathbb{Z}$ en $l$. La théorie de l'induction de Conlon $[4, \S 81 \mathrm{~B}]$ nous permet de donner une réponse complète.

Théorème 3. Soit q le cardinal de $\mathbb{F}$. Soit l un nombre premier. Si l ne divise pas $q^{2}-1$, alors le Théorème 2 reste vrai avec $\mathbb{Q}$ remplacé par $\mathbb{Z}_{(l)}$. Si l ne divise pas $q-1$, alors la partie (2) du Théorème 2 reste vraie avec $\mathbb{Q}$ remplacé par $\mathbb{Z}_{(l)}$.

Preuve. Soit $l$ premier. Un groupe fini $C$ est dit cyclique modulo $l$ si $C$ admet un quotient cyclique de noyau un $l$-groupe. Pour $H$ un groupe fini et $U, V \operatorname{deux} H$ ensembles finis la théorie de l'induction de Conlon implique que les $H$-modules $\mathbb{Z}_{(l)}[U]$ et $\mathbb{Z}_{(l)}[V]$ sont isomorphes si et seulement si pour tout sous-groupe $C$ cyclique modulo $l$ de $H$, les $C$-ensembles $U$ et $V$ sont isomorphes. Pour voir "seulement si" on applique (81.28) dans [4] avec $R=\mathbb{F}_{l}$, et pour "si" on utilise (81.25) avec $R=\mathbb{F}_{l}$, et les arguments dans (81.17) avec $R=\mathbb{Z}_{(l)}$.

Supposons que $l$ ne divise pas l'ordre de $G$. Alors les sous-groupes de $G$ cycliques modulo $l$ sont simplement les sous-groupes cycliques. Nous avons déjà vu que $X$ et $Y$, ainsi que $\bar{X}$ et $\bar{Y}$, sont $C$-isomorphes pour tout sous-groupe cyclique $C$ de $G$.

Considérons le cas où $l$ est la caractéristique de $\mathbb{F}$. Comme chaque élément dans $G$ d'ordre $l$ a une point fixe unique dans $\mathbb{P}^{1}(\mathbb{F})$, tout sous-groupe $H$ de $G$ cyclique modulo $l$ est soit cyclique soit un conjugué d'un sous-groupe de $B$. Lors de la preuve du Théorème 2 nous avons vu que $X$ et $Y$, ainsi que $\bar{X}$ et $\bar{Y}$, sont des $B$-ensembles isomorphes. Ceci termine la preuve pour ce $l$.

La dernière chose à montrer est que $\mathbb{Z}_{(l)}[\bar{X}] \cong \mathbb{Z}_{(l)}[\bar{Y}]$ en tant que $G$-modules pour les $l \neq 2$ divisant $q+1$. Supposons $l$ comme cela. Notons que $N^{\prime} / \mathbb{F}^{*}$ est un groupe diédrale d'ordre $2(q+1)$. Les $N^{\prime} / \mathbb{F}^{*}$-ensembles $\bar{X}$ et $\bar{Y}$ ont chacun un point fixe unique; notons $X^{\prime}$ et $Y^{\prime}$ les compléments. Soit $S$ un 2-sousgroupe de Sylow $N^{\prime} / \mathbb{F}^{*}$, et $H$ le sous-groupe maximal d'ordre impair de $N^{\prime} / \mathbb{F}^{*}$; ainsi $N^{\prime} / \mathbb{F}^{*}$ est le produit semi-direct de $H$ par $S$. Comme $\mathbb{Q}\left[X^{\prime}\right] \cong_{N^{\prime}} \mathbb{Q}\left[Y^{\prime}\right]$ on a $\mathbb{Q}\left[H \backslash X^{\prime}\right] \cong_{S} \mathbb{Q}\left[H \backslash Y^{\prime}\right]$. Comme $l$ ne divise pas $\# S$, on a $\mathbb{Z}_{(l)}\left[H \backslash X^{\prime}\right] \cong_{S}$ $\mathbb{Z}_{(l)}\left[H \backslash Y^{\prime}\right]$ par Conlon. Nous avons déjà vu que les stabilisateurs dans $T^{\prime} / \mathbb{F}^{*}$ des éléments de $X^{\prime}$ et de $Y^{\prime}$ sont d'ordre un ou deux. Donc les stabilisateurs dans $N^{\prime} / \mathbb{F}^{*}$ ont des conjugués dans $S$. Il en résulte que $X^{\prime}$ et $Y^{\prime}$ sont les induits des $S$-ensembles $H \backslash X^{\prime}$ et $H \backslash Y^{\prime}$ (cf. [4, §80B]). Par induction de modules, $\mathbb{Z}_{(l)}\left[X^{\prime}\right]$ et $\mathbb{Z}_{(l)}\left[Y^{\prime}\right]$ sont isomorphes en tant que $N^{\prime}$-modules. Par Conlon, $X^{\prime}$ et $Y^{\prime}$ sont isomorphes en tant que $C$-ensembles pour tout sous-groupe $C$ de $N^{\prime}$ cyclique 
modulo $l$. Mais tout sous-groupe cyclique modulo $l$ de $G$ est soit cyclique soit un conjugué d'un sous-groupe de $N^{\prime}$. Encore par Conlon, $\mathbb{Z}_{(l)}[\bar{X}] \cong_{G} \mathbb{Z}_{(l)}[\bar{Y}]$.

Remarque 4. Soit $l$ un nombre premier qui divise $q^{2}-1$. Il existe un sousgroupe $D$ de $N / \mathbb{F}^{*}$ ou de $N^{\prime} / \mathbb{F}^{*}$, diédral d'ordre $2 l$. Alors $D$ fixe plus de points dans $X$ que dans $Y$, et, si $l$ divise $q-1$, plus dans $\bar{X}$ que dans $\bar{Y}$. Par Conlon, on voit que pour la catégorie des $\mathbb{Z}_{(l)}$-modules les parties (1) et (2) du Théorème 2 sont vraies seulement si $l$ satisfait les conditions du Théorème 3 .

Théorème 4. Notations comme dans le Théorème 1. Soitl un nombre premier. Si $l$ ne divise pas $p^{2}-1$, alors il existe une isogénie $\operatorname{jac}(X / T) \rightarrow \operatorname{jac}\left(X / T^{\prime}\right) \times$ $\mathrm{jac}(X / B)^{2}$ de degré premier à $l$. Si $l$ ne divise pas $p-1$, alors il existe une isogénie $\mathrm{jac}(X / N) \rightarrow \mathrm{jac}\left(X / N^{\prime}\right) \times \mathrm{jac}(X / B)$ de degré premier à $l$.

Preuve. Nous notons d'abord que pour des objets $A$ et $B$ de $\mathcal{A}$ on a $\mathbb{Z}_{(l)} \otimes A \cong \mathbb{Z}_{(l)} \otimes B$ si et seulement s'il existe une isogénie $A \rightarrow B$ de degré premier à $l$. Sous les hypothèses faites sur $l$, le Théorème 3 donne l'existence d'isomorphismes $\left(\mathbb{Z}_{(l)} \otimes \operatorname{jac}(X)\right)^{T} \rightarrow\left(\mathbb{Z}_{(l)} \otimes \mathrm{jac}(X)\right)^{T^{\prime}} \oplus\left(\mathbb{Z}_{(l)} \otimes \mathrm{jac}(X)\right)^{B, 2}$ et $\left(\mathbb{Z}_{(l)} \otimes \mathrm{jac}(X)\right)^{N} \rightarrow\left(\mathbb{Z}_{(l)} \otimes \mathrm{jac}(X)\right)^{N^{\prime}} \oplus\left(\mathbb{Z}_{(l)} \otimes \mathrm{jac}(X)\right)^{B}$. Pour terminer, il suffit de voir que les noyaux des morphismes $\operatorname{jac}(X / H) \rightarrow \operatorname{jac}(X)$ sont d'ordre premier aux $l$ concernés, pour $H$ parmi $T, T^{\prime}, B, N$ et $N^{\prime}$. Mais ce noyau est le dual de Cartier du groupe du plus grand sous-revêtement étale abélien de $(X / H)_{\overline{\mathbb{Q}}}=Y /\left(H \cap \mathrm{SL}_{2}\left(\mathbb{F}_{p}\right)\right)$ dans $Y \rightarrow(X / H)_{\overline{\mathbb{Q}}}$, où $Y$ est une composante connexe de $X_{\overline{\mathbb{Q}}}$ (sur $\mathbb{C}$ cela se voit à l'aide des groupes fondamentaux). Pour $p<5$ on a $\operatorname{jac}(X)=0$, nous supposons donc que $p \geq 5$. Pour $N$ et $N^{\prime}$, les noyaux en question sont alors des 2-groupes. Pour $T$ et $B$ on obtient des quotients de $\mathbb{F}_{p}^{*}$, et $T^{\prime}$ donne un groupe cyclique d'ordre divisant $p+1$.

Remerciements. Nous tenons à remercier Laurent Moret-Bailly pour une lecture critique de ce texte.

\section{Références}

[1] A. Chambert-Loir and F. Sauvageot, Sur l'existence d'isogénies entre jacobiennes de courbes modulaires, manuscrit, version provisoire de décembre 1998; ainsi qu'une nouvelle version, voir http://www.math.jussieu.fr/ chambert/.

[2] I. Chen, The jacobians of non-split Cartan modular curves, Proc. London Math. Soc. (3) 77 (1998), 1-38.

[3] - On relations between jacobians of certain modular curves, A paraître dans Journal of Algebra, preprint 134, 3 septembre 1998, voir http://www.math.uiuc.edu/AlgebraicNumber-Theory.

[4] C.W. Curtis and I. Reiner, Methods of representation theory, Volume II, With applications to finite groups and orders, Pure and Applied Mathematics, John Wiley \& Sons, Inc., New York, 1994.

[5] H. Darmon and L. Merel, Winding quotients and some variants of Fermat's last theorem, J. Reine Angew. Math. 490 (1997), 81-100. 
[6] S.J. Edixhoven, On a result of Imin Chen, Prépublication 96-16 de l'IRMAR, Rennes, mai 1996.

[7] A. Grothendieck and J.A. Dieudonné, Eléments de géométrie algébrique I, Grundlehren Math. Wiss. 166, Springer-Verlag, 1971.

[8] E. Kani and M. Rosen, Idempotent relations and factors of Jacobians, Math. Ann. 284 (1989), 307-327.

[9] _ Idempotent relations among arithmetic invariants attached to number fields and algebraic varieties, J. Number Theory 46 (1994), 230-254.

[10] N.M. Katz and B. Mazur, Arithmetic moduli of elliptic curves. Annals of Mathematics Studies, 108, Princeton University Press, Princeton, NJ, 1985.

[11] B. Mazur, Modular curves and the Eisenstein ideal, Inst. Hautes Études Sci. Publ. Math. 47 (1977), 33-186.

[12] A.J. Scholl, Motives for modular forms, Invent. Math. 100 (1990), 419-430.

[13] _ Classical motives, Proc. Sympos. Pure Math. 55 (1994), 163-187.

[14] J.-P. Serre, Représentations linéaires des groupes finis, (3ème édition corrigée), Hermann, Paris, 1978.

Mathematisch Instituut, Universiteit Leiden, Postbus 9512, 2300 RA Leiden, PaysBAS

E-mail address: desmit@math.leidenuniv.nl

IRMAR, Université de Rennes 1, 35042 Rennes Cedex, FRAnCE

E-mail address: edix@maths.univ-rennes1.fr 